\title{
A NOTE ON THE COMPUTATION OF ALL ZEROS OF SIMPLE QUATERNIONIC POLYNOMIALS*
}

\author{
DRAHOSLAVA JANOVSKÁ† AND GERHARD OPFER ${ }^{\ddagger}$
}

\begin{abstract}
Polynomials with quaternionic coefficients located on only one side of the powers (we call them simple polynomials) may have two different types of zeros: isolated and spherical zeros. We will give a new characterization of the types of the zeros and, based on this characterization, we will present an algorithm for producing all zeros including their types without using an iteration process which requires convergence. The main tool is the representation of the powers of a quaternion as a real, linear combination of the quaternion and the number one (as introduced by Pogorui and Shapiro [Complex Var. and Elliptic Funct., 49 (2004), pp. 379-389]) and the use of a real companion polynomial which already was introduced for the first time by Niven [Amer. Math. Monthly, 48 (1941), pp. 654-661]. There are several examples.
\end{abstract}

Key words. zeros of quaternionic polynomials, structure of zeros of quaternionic polynomials

AMS subject classifications. 11R52, 12E15, 12Y05, 65H05

DOI. $10.1137 / 090748871$

1. Introduction. The first attempts to find the zeros of a quaternionic polynomial were made by Niven in 1941 [13]. Polynomials of type (1.3) (see below), which we shall call simple, were considered. Niven's idea was to divide the polynomial by a quadratic polynomial with (certain) real coefficients and to adjust the coefficients of the quadratic polynomial by an iterative procedure in such a way that the remainder of the division vanished. Finally, it was shown that the set of zeros of the resulting quadratic polynomial also contained quaternions. The first numerically working algorithm based on these ideas was presented in 2001 by Serôdio, Pereira, and Vitória [17]. Further contributions to polynomials with quaternionic coefficients were made by Pumplün and Walcher (2002) [16], De Leo, Ducati, and Leonardi (2006) [12], Gentili and Struppa (2007) [2], Gentili, Struppa, and Vlacci (2008) [3], and Gentili and Stoppato (2008) [4]. Polynomials over division rings were investigated by Gordon and Motzkin (1965) [5]. See also the book by Lam [10, section 16]. A large bibliography on quaternions in general was given by Gsponer and Hurni (2006) [6]. We would also like to mention an extension of this investigation to polynomials with coefficients at either side of the powers. See Janovská and Opfer [7]. Only as an aperçu we mention that Felix Klein apparently was not so fond of quaternions. He wrote [9, p. 20], "Daß man in dieser Theorie zu Resultaten gelangt, die im Sinne der gewöhnlichen Algebra absurd sind, zeigt folgendes Beispiel:...". ${ }^{1}$ And then a polynomial of degree three with infinitely many zeros follows.

Another successful idea was introduced by Pogorui and Shapiro (2004) [15]. They systematically used the fact that a power of a quaternion $z$ could be represented in the

* Received by the editors February 6, 2009; accepted for publication (in revised form) January 15, 2010; published electronically April 2, 2010. This research was supported by the research project MSM 6046137306 of The Ministry of Education, Youth and Sports, Czech Republic.

http://www.siam.org/journals/sinum/48-1/74887.html

${ }^{\dagger}$ Department of Mathematics, Institute of Chemical Technology, Prague, Technická 5, 16628 Prague 6, Czech Republic (janovskd@vscht.cz).

${ }^{\ddagger}$ Faculty for Mathematics, Informatics, and Natural Sciences (MIN), University of Hamburg, Bundestraße 55, 20146 Hamburg, Germany (opfer@math.uni-hamburg.de).

1 "That in this theory one obtains results which are absurd in the sense of ordinary algebra, shows the following example:...." 
form $z^{j}=\alpha z+\beta$, where $\alpha, \beta$ were real and where $\alpha, \beta$ did not fully depend on $z$ but only on the real part (the first component) and the length of $z$ (as a vector in $\mathbb{R}^{4}$ ). The emphasis of the work by Pogorui and Shapiro was put mainly on the structure of the set of zeros, in particular on the number of zeros, but not on the systematic computation of the zeros. They use the multiplicities of the zeros of a certain real polynomial as a means for characterizing the two types of zeros which will emerge for simple, quaternionic polynomials. This real polynomial is associated with the given, simple, quaternionic polynomial and will be called companion polynomial in this investigation. The characterization of the two types of zeros presented here is based, however, on the value of a certain quaternionic number. One type is characterized by the value zero, the other type by any nonzero value. We do not use the multiplicities. Based on this new characterization, an algorithm is presented for finding all zeros including the type of zero. It is based on the (real and complex) zeros of the real companion polynomial. The resulting algorithm is simple. It was tested successfully on hundreds of examples. A summary of the algorithm is given at the end of the paper.

By $\mathbb{R}, \mathbb{C}$ we denote the fields of real and complex numbers, respectively, and by $\mathbb{Z}$ the set of integers. By $\mathbb{H}$ we denote the (skew) field of quaternions that consists of elements of $\mathbb{R}^{4}$, equipped with the multiplication rule

$$
\begin{aligned}
a b:= & \left(a_{1} b_{1}-a_{2} b_{2}-a_{3} b_{3}-a_{4} b_{4}, a_{1} b_{2}+a_{2} b_{1}+a_{3} b_{4}-a_{4} b_{3},\right. \\
& \left.a_{1} b_{3}-a_{2} b_{4}+a_{3} b_{1}+a_{4} b_{2}, a_{1} b_{4}+a_{2} b_{3}-a_{3} b_{2}+a_{4} b_{1}\right),
\end{aligned}
$$

where $a:=\left(a_{1}, a_{2}, a_{3}, a_{4}\right), b:=\left(b_{1}, b_{2}, b_{3}, b_{4}\right), a_{j}, b_{j} \in \mathbb{R}, j=1,2,3,4$. By $\Re a$ we will denote the real part of $a$, which is defined by $a_{1}$, the first component of $a$. By $\Im a$, we denote the imaginary part, the second component $a_{2}$ of $a$, and $|a|$ denotes the absolute value of $a$, where $|a|:=\sqrt{a_{1}^{2}+a_{2}^{2}+a_{3}^{2}+a_{4}^{2}}$ and where $a:=\left(a_{1}, a_{2}, a_{3}, a_{4}\right)$ in all cases. The multiplication rule implies, in particular,

$$
\Re(a b)=\Re(b a) \text { and } r a=a r \text { for } a, b \in \mathbb{H}, r \in \mathbb{R} .
$$

Let

$$
p_{n}(z):=\sum_{j=0}^{n} a_{j} z^{j}, \quad z, a_{j} \in \mathbb{H}, \quad j=0,1,2, \ldots, n, \quad a_{0}, a_{n} \neq 0
$$

be a given quaternionic polynomial with degree $n$ where $n$ is a positive integer. As we already have mentioned, such a polynomial will be called simple. We are interested in finding its zeros. The assumption $a_{0} \neq 0$ implies that the origin is never a zero of $p_{n}$. The assumption $a_{n} \neq 0$ ensures that the degree of the polynomial is not less than $n$. Without loss of generality we could assume $a_{n}=1$. It should be noted that the general form of a quaternionic monomial would be $a_{0} \cdot z \cdot a_{1} \cdot z \cdot a_{2} \cdots a_{j-1} \cdot z \cdot a_{j}$ such that the above $p_{n}$ is only a very special type of quaternionic polynomial. See [14] for some statements on polynomials of general type. It also should be noted that it is still possible to evaluate $p_{n}(z)$ by Horner's scheme, although coefficients and argument are in $\mathbb{H}$.

By looking at

$$
p_{2}(z):=z^{2}+1
$$

we see that not only $z_{1,2}:= \pm \mathbf{i}$ are zeros of $p_{2}$, but also $h^{-1} z_{1,2} h$ for all $h \in \mathbb{H} \backslash\{0\}$. In general, if $p_{n}$ is a polynomial with real coefficients and $z_{0}$ is a zero of $p_{n}$, then $h^{-1} z_{0} h$ 
is also a zero for all $h \in \mathbb{H} \backslash\{0\}$. This follows from $h^{-1} p_{n}(z) h=p_{n}\left(h^{-1} z h\right)$. Since $h^{-1} z h=z$ for real $z$, we obtain new zeros only if $z$ is not real. Only in passing we note that the above $p_{2}$ differs from $\tilde{p}_{2}$ defined by $\tilde{p}_{2}(z):=(z-\mathbf{i})(z+\mathbf{i})$, and $\tilde{p}_{2}$ does not belong in the class of simple polynomials defined in (1.3). The properties of $p_{2}$ lead to the introduction of equivalence classes of quaternions. ${ }^{2}$

Definition 1.1. Two quaternions $a, b \in \mathbb{H}$ are called equivalent, denoted by $a \sim b$, if

$$
a \sim b \Leftrightarrow \exists h \in \mathbb{H} \backslash\{0\} \text { such that } a=h^{-1} b h .
$$

The set

$$
[a]:=\left\{u \in \mathbb{H}: u=h^{-1} \text { ah for all } h \in \mathbb{H} \backslash\{0\}\right\}
$$

will be called an equivalence class of a.

It is easily seen that $\sim$ indeed defines an equivalence relation. Equivalent quaternions $a, b$ can be easily recognized by

$$
a \sim b \Leftrightarrow \Re a=\Re b \text { and }|a|=|b| \text { (cf. [8]). }
$$

We identify a real number $a_{1}$ by the quaternion $\left(a_{1}, 0,0,0\right)$ and a complex number $a_{1}+\mathbf{i} a_{2}$ by the quaternion $\left(a_{1}, a_{2}, 0,0\right)$. Let $a$ be real. Then $[a]=\{a\}$, which means that in this case, the equivalence class consists only of one element, $a$. If $a$ is not real, then $[a]$ contains infinitely many elements, which according to (1.5), (1.6), (1.7) can be characterized by

$$
[a]:=\{z \in \mathbb{H}: \Re z=\Re a,|z|=|a|\}
$$

and can be regarded as a two dimensional sphere in $\mathbb{R}^{4}$. Let $a:=\left(a_{1}, a_{2}, a_{3}, a_{4}\right) \in \mathbb{H}$. Then, the conjugate of $a$, denoted by $\bar{a}$, is defined by

$$
\bar{a}:=\left(a_{1},-a_{2},-a_{3},-a_{4}\right) .
$$

From (1.8) it follows that

$$
\bar{a} \in[a] .
$$

The most important rule for the conjugate is

$$
\overline{a b}=\bar{b} \bar{a} .
$$

And for the inverse, there is the formula

$$
a^{-1}=\frac{\bar{a}}{|a|^{2}} \text { for } a \neq 0 .
$$

2. Isolated and spherical zeros of polynomials. The set of zeros of a polynomial of type (1.3) will separate into two classes. This is the main content of this section.

Definition 2.1. Let $z_{0}$ be a zero of $p_{n}$, where $p_{n}$ is defined in (1.3). If $z_{0}$ is not real and has the property that $p_{n}(z)=0$ for all $z \in\left[z_{0}\right]$, then we will say that $z_{0}$ will generate a spherical zero. For short, we will also say that $z_{0}$ is, rather than

\footnotetext{
${ }^{2}$ Algebraists use the phrase conjugacy classes.
} 
generates, a spherical zero. If $z_{0}$ is real or does not generate a spherical zero, it is called an isolated zero. The number of zeros of $p_{n}$ will be defined as the number of equivalence classes, which contain at least one zero of $p_{n}$.

In what follows, we will see that under the assumption that $z_{0}$ is a zero of $p_{n}$, either all elements in $\left[z_{0}\right]$ are zeros, or $z_{0}$ is the only zero in $\left[z_{0}\right]$. For examples, look back at the remarks in connection with the polynomial defined in (1.4). One of the results of Pogorui and Shapiro is that the number of zeros does not exceed $n$. However, this result already was known to Gordon and Motzkin (1965) Theorem 2, [5]. A result by Eilenberg and Niven (1944) [1] says that all simple polynomials $p_{n}$ of degree $n \geq 1$ have at least one zero. Actually, the result by Eilenberg and Niven applies to all quaternionic polynomials which contain only one monomial with the highest degree.

All powers $z^{j}, j \in \mathbb{Z}$ of a quaternion $z$ have the form $z^{j}=\alpha z+\beta$ with real $\alpha, \beta$. This was used in the context of quaternionic polynomials for the first time by Pogorui and Shapiro [15]. In particular,

$$
z^{2}=2 \Re z z-|z|^{2} .
$$

In order to determine the numbers $\alpha, \beta$, we set up the following iteration (for negative $j$ and nonvanishing $z$ we use $z^{-1}=\frac{\bar{z}}{|z|^{2}}$ instead of $z$ ):

$$
\begin{aligned}
z^{j} & =\alpha_{j} z+\beta_{j}, \quad \alpha_{j}, \beta_{j} \in \mathbb{R}, \quad j=0,1, \ldots, \text { where } \\
\alpha_{0} & =0, \quad \beta_{0}=1, \\
\alpha_{j+1} & =2 \Re z \alpha_{j}+\beta_{j}, \\
\beta_{j+1} & =-|z|^{2} \alpha_{j}, \quad j=0,1, \ldots
\end{aligned}
$$

The corresponding iteration given by Pogorui and Shapiro is a three term recursion, whereas this one (formulas (2.3) to (2.5)) is a two term recursion. Thus, they differ formally. In some cases, two term recursions are more stable than the corresponding three term recursion. For an example, see Laurie (1999) [11]. The given recursion is a very economical means to calculate the powers of a quaternion. In order to compute all powers of $z \in \mathbb{H}$ up to degree $n$ by standard means, one needs $n-1$ quaternionic multiplications, where one quaternionic multiplication (see (1.1)) needs 28 flops (real floating point operations), whereas the recursion (2.3) to (2.5) needs only $3 n$ flops. The sequence $\left\{\alpha_{j}\right\}$ is defined by a difference equation of order two with constant coefficients. Using the theory of difference equations, it is possible to give a closed form solution for $\alpha_{j}$. There are two versions valid for the case $z \notin \mathbb{R}$. One of the versions is purely real; the other is formally complex. The real version of the solution is as follows:

$$
\alpha_{j}=\frac{\Im\left\{u_{1}^{j}\right\}}{\sqrt{|z|^{2}-(\Re z)^{2}}}, u_{1}:=\Re z+\mathbf{i} \sqrt{|z|^{2}-(\Re z)^{2}}, \sqrt{|z|^{2}-(\Re z)^{2}}>0, j \geq 0,
$$

where $u_{1}$ is one of the two complex solutions of $u^{2}-2 \Re z u+|z|^{2}=0$. Formula (2.6) for $\alpha_{j}$ is easier to program than the iteration (2.3) to (2.5). However, since a power is involved, an economical use of (2.6) would also require an iteration.

By means of (2.2) the polynomial $p_{n}$ can be written as

$$
p_{n}(z):=\sum_{j=0}^{n} a_{j} z^{j}=\sum_{j=0}^{n} a_{j}\left(\alpha_{j} z+\beta_{j}\right)=\left(\sum_{j=0}^{n} \alpha_{j} a_{j}\right) z+\sum_{j=0}^{n} \beta_{j} a_{j}=: A(z) z+B(z) .
$$


Theorem 2.2. Let $z_{0} \in \mathbb{H}$ be fixed. Then $A(z)=$ const, $B(z)=$ const for all $z \in\left[z_{0}\right]$, where $A, B$ are defined in (2.7). Let $z_{0}$ be a zero of $p_{n}$. Then,

$$
p_{n}\left(z_{0}\right)=A(z) z_{0}+B(z)=0 \text { for all } z \in\left[z_{0}\right]
$$

The quantities $A, B$ in (2.8) can only vanish simultaneously. If $A\left(z_{0}\right)=0$ and if $z_{0}$ is not real, then $z_{0}$ generates a spherical zero of $p_{n}$. If $A\left(z_{0}\right) \neq 0$, then $z_{0}$ is an isolated zero of $p_{n}$.

Proof. From (2.3) to (2.5) it is clear that the coefficients $\alpha_{j}, \beta_{j}, j \geq 0$ are the same for all $z$ with the same $\Re z,|z|$. Thus, the coefficients are the same for all $z \in\left[z_{0}\right]$ therefore, $A(z)=$ const, $B(z)=$ const for all $z \in\left[z_{0}\right]$. If $A\left(z_{0}\right)=0$, then necessarily $B\left(z_{0}\right)=0$ and vice versa. Recall that $z_{0} \neq 0$. If $A\left(z_{0}\right)=0$, we have $p(z)=0$ for all $z \in\left[z_{0}\right]$. This implies that $z_{0}$ generates a spherical zero if $z_{0}$ is not real. Let $A\left(z_{0}\right) \neq 0$. Then, for all $z \in\left[z_{0}\right](2.8)$ defines $z_{0}$ uniquely. Apart from $z_{0}$, there is no zero in $\left[z_{0}\right]$.

From here on, it seems reasonable to change the notation from $A(z)$ to $A(\Re z,|z|)$ and from $B(z)$ to $B(\Re z,|z|)$ if the arguments should be mentioned at all. For the following theorem, see also Gordon and Motzkin, Theorem 4 [5].

TheOrem 2.3. Let $z_{0}, z_{1} \in \mathbb{H}$ be two different zeros of $p_{n}$ with $z_{0} \in\left[z_{1}\right]$. Then $p_{n}(z)=0$ for all $z \in\left[z_{1}\right]$ and $z_{0}$ generates a spherical zero of $p_{n}$, and $A(\Re z,|z|)=$ $B(\Re z,|z|)=0$, where $A, B$ are defined in (2.7).

Proof. Since $z_{0}, z_{1}$ are assumed to be different and to belong to the same equivalence class, they cannot be real. It follows from $(2.7)$ that $p_{n}\left(z_{j}\right)=A(\Re z,|z|) z_{j}+$ $B(\Re z,|z|)=0$ for all $z \in\left[z_{0}\right]=\left[z_{1}\right], j=0,1$. Taking differences, we obtain $p_{n}\left(z_{0}\right)-p_{n}\left(z_{1}\right)=A(\Re z,|z|)\left(z_{0}-z_{1}\right)=0$ for all $z \in\left[z_{1}\right]=\left[z_{0}\right]$, implying $A(\Re z,|z|)=0$. According to Theorem 2.2, the zero $z_{0}$ generates a spherical zero of $p_{n}$.

This shows that Definition 2.1 is meaningful. Either, with $z \notin \mathbb{R}$, the whole equivalence class $[z]$ consists of zeros ( $z$ is a spherical zero), or apart from $z \in \mathbb{H}$, there is no zero in $[z]$ ( $z$ is an isolated zero).

Thus, we have the following classification of the zeros $z_{0}$ of $p_{n}$ given in (1.3):

1. $z_{0}$ is real. By definition, $z_{0}$ is isolated.

2. $z_{0}$ is not real. $A\left(\Re z_{0},\left|z_{0}\right|\right)=0 \Rightarrow z_{0}$ is spherical; all $z \in\left[z_{0}\right]$ are zeros of $p_{n}$.

3. $z_{0}$ is not real. $A\left(\Re z_{0},\left|z_{0}\right|\right) \neq 0 \Rightarrow z_{0}$ is isolated.

3. The companion polynomial. Let $p_{n}$ be the polynomial defined in (1.3) with the quaternionic coefficients $a_{0}, a_{1}, \ldots, a_{n}$. Following Niven [13, section 2] or more recently, Pogorui and Shapiro [15], we define the polynomial $q_{2 n}$ of degree $2 n$ with real coefficients by

$$
\begin{aligned}
q_{2 n}(z) & :=\sum_{j, k=0}^{n} \overline{a_{j}} a_{k} z^{j+k}=\sum_{k=0}^{2 n} b_{k} z^{k}, \quad z \in \mathbb{C}, \text { where } \\
b_{k} & :=\sum_{j=\max (0, k-n)}^{\min (k, n)} \overline{a_{j}} a_{k-j} \in \mathbb{R}, \quad k=0,1, \ldots, 2 n .
\end{aligned}
$$

We will call $q_{2 n}$ the companion polynomial of the quaternionic polynomial $p_{n}$. It always should be regarded as a polynomial over $\mathbb{C}$, not over $\mathbb{H}$. Since it has real coefficients, we may assume that it is always possible to find all (real and complex) zeros of $q_{2 n}$. How are the quaternionic zeros of $p_{n}$ related to the real or complex zeros of $q_{2 n}$ ? This question will be answered in this section. 
Lemma 3.1. Let $p_{n}(z)=A(\Re z,|z|) z+B(\Re z,|z|)$ be as described in (2.7). Then, (we delete the arguments of $A$ and $B$ )

$$
q_{2 n}(z)=|A|^{2} z^{2}+2 \Re(\bar{A} B) z+|B|^{2} .
$$

Proof. Let $z^{j}=\alpha_{j} z+\beta_{j}$, cf. (2.2) to (2.5). Then, we have

$$
\begin{aligned}
q_{2 n}(z) & =\sum_{j, k=0}^{n} \overline{a_{j}} a_{k} z^{j+k}=\sum_{j=0}^{n} \overline{a_{j}}\left(\sum_{k=0}^{n} a_{k} z^{k}\right) z^{j}=\sum_{j=0}^{n} \overline{a_{j}}(A z+B) z^{j} \\
& =\sum_{j=0}^{n} \overline{a_{j}}(A z+B)\left(\alpha_{j} z+\beta_{j}\right) \quad\left[\alpha_{j}, \beta_{j} \in \mathbb{R}\right] \\
& =\sum_{j=0}^{n}\left(\alpha_{j} \overline{a_{j}}\right) A z^{2}+\sum_{j=0}^{n}\left(\beta_{j} \overline{a_{j}}\right) A z+\sum_{j=0}^{n}\left(\alpha_{j} \overline{a_{j}}\right) B z+\sum_{j=0}^{n}\left(\beta_{j} \overline{a_{j}}\right) B \\
& =|A|^{2} z^{2}+2 \Re(\bar{A} B) z+|B|^{2} .
\end{aligned}
$$

Thus, the formula (3.3) is correct.

Formula (3.3) again shows that $A(\Re z,|z|)=0 \Leftrightarrow B(\Re z,|z|)=0$ if $z$ is a zero of $p_{n}$. The real zeros of $p_{n}$ can be discovered quite easily.

Theorem 3.2. Let $z_{0} \in \mathbb{R}$. Then,

$$
q_{2 n}\left(z_{0}\right)=0 \Leftrightarrow p_{n}\left(z_{0}\right)=0 .
$$

The set of the real zeros is the same for $p_{n}$ and for $q_{2 n}$.

Proof. On the real line $z \in \mathbb{R}$, we have $q_{2 n}(z)=\left|p_{n}(z)\right|^{2}$.

Since $q_{2 n}$ has real coefficients and because of $q_{2 n}(z)=\left|p_{n}(z)\right|^{2}$ for $z \in \mathbb{R}$, the zeros of $q_{2 n}$ come always in pairs

$$
\ldots r, r, \ldots, a+\mathbf{i} b, a-\mathbf{i} b, \ldots
$$

where $r, a, b$ represent real numbers.

The case of spherical zeros is easy as well.

TheOREM 3.3. Let $z_{0}$ be a nonreal zero of $q_{2 n}$ and let $A\left(\Re z_{0},\left|z_{0}\right|\right)=0$. See (2.7) for the definition of the quaternion $A$. Then, $z_{0}$ generates a spherical zero of $p_{n}$.

Proof. Equation (3.3) implies that $B\left(\Re z_{0},\left|z_{0}\right|\right)=0$ as well, where the quaternion $B$ is also defined in (2.7). Thus, $p_{n}\left(z_{0}\right)=0$ by (2.7), and from Theorem 2.2 we conclude that $z_{0}$ generates a spherical zero of $p_{n}$.

For the remaining part, we have to investigate those nonreal zeros $z$ of $q_{2 n}$ for which $A(\Re z,|z|) \neq 0$. In general, we will have $p_{n}(z) \neq 0$. However, we can try to find a $z_{0} \in[z]$ such that $p_{n}\left(z_{0}\right)=0$. If that is possible, $z_{0}$ necessarily must have the form

$$
z_{0}:=-A(\Re z,|z|)^{-1} B(\Re z,|z|)=-\frac{\overline{A(\Re z,|z|)} B(\Re z,|z|)}{|A(\Re z,|z|)|^{2}} .
$$

This follows from Theorem 2.2 and formulas (1.9) and (2.7). We have to show that $z_{0} \in[z]$, which means that we have to show that $\Re z_{0}=\Re z$ and $\left|z_{0}\right|=|z|$.

Lemma 3.4. Let $z$ be a nonreal zero of $q_{2 n}$ with $A(\Re z,|z|) \neq 0$. Define $z_{0}$ as in (3.5). Then

$$
\Re z_{0}=\Re z \text { and }\left|z_{0}\right|=|z| .
$$


Proof. According to Lemma 3.1, the zero $z$ of $q_{2 n}$ obeys the equation

$$
q_{2 n}(z)=|A(\Re z,|z|)|^{2} z^{2}+2 \Re(\overline{A(\Re z,|z|)} B(\Re z,|z|)) z+|B(\Re z,|z|)|^{2}=0 .
$$

From here on, we delete the arguments of $A$ and $B$. We put

$$
\left(z_{1}, z_{2}, 0,0\right):=z ; \quad\left(v_{1}, v_{2}, v_{3}, v_{4}\right):=\bar{A} B .
$$

Then, by separating the real and imaginary parts, $\left(3.3^{\prime}\right)$ implies

$$
|A|^{2}\left(z_{1}^{2}-z_{2}^{2}\right)+2 v_{1} z_{1}+|B|^{2}=0, \quad|A|^{2} z_{1}+v_{1}=0 .
$$

It follows from the definition of $z_{0}$ that

$$
\Re z_{0}=-\frac{\Re(\bar{A} B)}{|A|^{2}}=-\frac{v_{1}}{|A|^{2}}=z_{1}=\Re z,
$$

where the last equation follows from the second equation in (3.7). Moreover,

$$
\left|z_{0}\right|=\left|-\frac{\bar{A} B}{|A|^{2}}\right|=\frac{|B|}{|A|} .
$$

If we insert the second equation of (3.7) into the first one, we obtain

$$
-|A|^{2}\left(z_{1}^{2}+z_{2}^{2}\right)+|B|^{2}=0
$$

and this gives the desired property $\frac{|B|^{2}}{|A|^{2}}=|z|^{2}$, and thus, $\left|z_{0}\right|=|z|$.

THEOREM 3.5. Let $p_{n}$ be given, and let $q_{2 n}$ be the corresponding companion polynomial, and assume that $z$ is a nonreal, complex zero of $q_{2 n}$ with $A(\Re z,|z|) \neq 0$. Then, $z_{0}$ defined in formula (3.5) is an isolated zero of $p_{n}$. If we use the notation (3.6) and $|v|=\sqrt{v_{2}^{2}+v_{3}^{2}+v_{4}^{2}}$, we can give $z_{0}$ also the following form, denoted for the moment by

$$
Z_{0}:=\left(z_{1},-\frac{\left|z_{2}\right|}{|v|} v_{2},-\frac{\left|z_{2}\right|}{|v|} v_{3},-\frac{\left|z_{2}\right|}{|v|} v_{4}\right) .
$$

Proof. We will show that $Z_{0}=z_{0}$. Clearly, we have $Z_{0} \in[z]$. For an arbitrary $a \in \mathbb{H}$ let us denote by $\operatorname{vec}(a)$ the three dimensional vector consisting of the last three components of $a$. From the previous lemma we know that $\left|\operatorname{vec}\left(z_{0}\right)\right|=\frac{|v|}{|A|^{2}}=\left|z_{2}\right|$, thus,

$$
\frac{1}{|A|^{2}}=\frac{\left|z_{2}\right|}{|v|}
$$

In the formula for $Z_{0}$ we replace the quantity $\frac{\left|z_{2}\right|}{|v|}$ by $\frac{1}{|A|^{2}}$, and we obtain $Z_{0}=z_{0}$.

With respect to (3.5), formula (3.8) has the advantage that it involves only the product $\bar{A} B$. Formula (3.5) also needs $|A|^{2}$.

There is still one missing link. Is it true that the zeros of the companion polynomial $q_{2 n}$ really exhaust all zeros of $p_{n}$, or is it possible that $p_{n}$ has a zero which we do not find by checking all zeros of $q_{2 n}$ ?

THEOREM 3.6. Let $p_{n}\left(z_{0}\right)=0$, where $p_{n}$ is defined in (1.3). Then, there is an $z \in \mathbb{C}$ with $z \in\left[z_{0}\right]$ such that $q_{2 n}(z)=0$, where $q_{2 n}$ is defined in (3.1), (3.2). 
Proof. If $z_{0} \in \mathbb{R}$, we have $q_{2 n}\left(z_{0}\right)=0$. This follows from Theorem 3.2. If $A\left(\Re z_{0},\left|z_{0}\right|\right)=0$ and $z_{0}$ is not real, then the class $\left[z_{0}\right]$ contains exactly one complex $z$ with a positive imaginary part such that $q_{2 n}(z)=0$. From here on, we assume that $A\left(\Re z_{0},\left|z_{0}\right|\right) \neq 0$. We have $p_{n}\left(z_{0}\right)=A\left(\Re z_{0},\left|z_{0}\right|\right) z_{0}+B\left(\Re z_{0},\left|z_{0}\right|\right)=0$, and thus

$$
z_{0}=-\frac{\overline{A\left(\Re z_{0},\left|z_{0}\right|\right)} B\left(\Re z_{0},\left|z_{0}\right|\right)}{\left|A\left(\Re z_{0},\left|z_{0}\right|\right)\right|^{2}} .
$$

For $q_{2 n}$ we have the formula (3.3), which is a quadratic equation with real coefficients, and one of the two complex zeros is (we delete the arguments of $A, B$ )

$$
z=-\frac{\Re(\bar{A} B)}{|A|^{2}}+\frac{\mathbf{i}}{|A|^{2}} \sqrt{|A|^{2}|B|^{2}-(\Re(\bar{A} B))^{2}} .
$$

Since $|\Re u| \leq|u|$ for all $u \in \mathbb{H}$, the radicand in (3.10) is never negative. It remains to show that $z \in\left[z_{0}\right]$, which is equivalent to $\Re z_{0}=\Re z$ and $\left|z_{0}\right|^{2}=|z|^{2}$. From (3.9) and (3.10) we deduce that

$$
\Re z=-\frac{\Re(\bar{A} B)}{|A|^{2}}=\Re z_{0} .
$$

From the same equations we obtain

$$
|z|^{2}=\frac{(\Re(\bar{A} B))^{2}}{|A|^{4}}+\frac{|A|^{2}|B|^{2}-(\Re(\bar{A} B))^{2}}{|A|^{4}}=\frac{|B|^{2}}{|A|^{2}}=\left|z_{0}\right|^{2} .
$$

Conclusion 3.7. The proposed procedure finds all zeros of the quaternionic polynomial $p_{n}$ (defined in (1.3)). The set of zeros of $p_{n}$ is not empty, and the number of zeros (see Definition 2.1) does not exceed $n$.

The following example shows all typical features of a quaternionic polynomial.

Example 3.8. Let

$$
p_{6}(z):=z^{6}+\mathbf{j} z^{5}+\mathbf{i} z^{4}-z^{2}-\mathbf{j} z-\mathbf{i} .
$$

Then, the companion polynomial for $p_{6}$ is

$$
q_{12}(x)=x^{12}+x^{10}-x^{8}-2 x^{6}-x^{4}+x^{2}+1 .
$$

The 12 zeros of $q_{12}$ are

$$
1 \text { (twice), } \quad-1 \text { (twice), } \quad \pm \mathbf{i} \text { (twice each), } \quad 0.5( \pm 1 \pm \mathbf{i}) .
$$

There are two different real zeros $z_{1,2}= \pm 1$ which are also zeros of $p_{6}$. There is one spherical zero $z_{3}=\mathbf{i}$ of $p_{6}$ ( $-\mathbf{i}$ generates the same spherical zero). And finally there are two isolated zeros which have to be computed from $x=0.5( \pm 1 \pm \mathbf{i})$ by formula (3.8). This formula yields

$$
z_{4}:=0.5(1,-1,-1,-1), \quad z_{5}:=0.5(-1,1,-1,-1),
$$

and $p_{6}$ has altogether five zeros in the sense of Definition 2.1. 
4. Polynomials with coefficients on the right side of the powers. If we want to compute the zeros of

$$
\tilde{p}_{n}(z):=\sum_{j=0}^{n} z^{j} a_{j}, \quad z, a_{j} \in \mathbb{H}, j=0,1,2, \ldots, n, \quad a_{0}, a_{n} \neq 0,
$$

rather than those of $p_{n}$, we apply the former theory to

$$
p_{n}(z):=\overline{\tilde{p}_{n}(\bar{z})}=\sum_{j=0}^{n} \overline{a_{j}} z^{j}, \quad z, a_{j} \in \mathbb{H}, j=0,1,2, \ldots, n, \quad a_{0}, a_{n} \neq 0 .
$$

The companion polynomial $q_{2 n}$ is identical for $\tilde{p}_{n}$ and for $p_{n}$, and thus, the zeros of the companion polynomials are the same.

LEMma 4.1. The two polynomials $\tilde{p}_{n}(z):=\sum_{j=0}^{n} z^{j} a_{j}$ and $p_{n}(z):=\sum_{j=0}^{n} \overline{a_{j}} z^{j}$ have the same real and spherical zeros. And for nonreal isolated zeros we have

$$
p_{n}(z)=0 \Longleftrightarrow \tilde{p}_{n}(\bar{z})=0 .
$$

Proof. An adaption of the theory of the foregoing section.

5. Numerical considerations. The polynomial in Example 3.8 is a contrived example. It has the property that $p_{6}(z)=\left(z^{2}+\mathbf{j} z+\mathbf{i}\right)\left(z^{4}-1\right)$. Normally, one is not able to guess the zeros, and one has to rely on machine computations. If we compute the zeros of $q_{12}$ of the previous example given in (3.12), we find by MATLAB computation the figures listed in Table 1, which are not as precise as desired though the integer coefficients of $p_{12}$ are exact.

There is the following remark. The four zeros with multiplicity one, numbered 3, 4, 7, 8 in Table 1, are precise to machine precision; however, all other zeros, which are zeros with multiplicity 2 , have errors of magnitude $10^{-8}$. It is easy to improve on these zeros. If $z$ is one of the zeros with multiplicity 2 , an application of one step of Newton's method applied to $q_{2 n}^{\prime}=0$ with starting point $z$ is sufficient to obtain machine precision. For zeros of multiplicity 4, one should apply Newton's method to $q_{2 n}^{\prime \prime \prime}=0$, etc., possibly with two steps.

We made some hundred tests with polynomials $p_{n}$ of degree $n \leq 50$ with random integer coefficients in the range $[-5,5]$ and with real coefficients in the range $[0,1]$. In all cases we found only (nonreal) isolated zeros $z$. The test cases showed $\left|p_{n}(z)\right| \approx$

TABLE 1

Zeros of $q_{12}$ by MATLAB computations and correct values.

\begin{tabular}{r|rr|c|}
1 & -1.00000000000000 & $+0.00000001131891 \mathbf{i}$ & -1 \\
2 & -1.00000000000000 & $-0.00000001131891 \mathbf{i}$ & -1 \\
3 & -0.50000000000000 & $+0.86602540378444 \mathbf{i}$ & $0.5(-1+\sqrt{3} \mathbf{i})$ \\
4 & -0.50000000000000 & $-0.86602540378444 \mathbf{i}$ & $0.5(-1-\sqrt{3} \mathbf{i})$ \\
5 & 1.00000000000000 & $+0.00000001376350 \mathbf{i}$ & 1 \\
6 & 1.00000000000000 & $-0.00000001376350 \mathbf{i}$ & 1 \\
7 & 0.50000000000000 & $+0.86602540378444 \mathbf{i}$ & $0.5(1+\sqrt{3} \mathbf{i})$ \\
8 & 0.50000000000000 & $-0.86602540378444 \mathbf{i}$ & $0.5(1-\sqrt{3} \mathbf{i})$ \\
9 & 0.00000000001566 & $+1.00000000619055 \mathbf{i}$ & $\mathbf{i}$ \\
10 & 0.00000000001566 & $-1.00000000619055 \mathbf{i}$ & $-\mathbf{i}$ \\
11 & -0.00000000001566 & $+0.99999999380945 \mathbf{i}$ & $\mathbf{i}$ \\
12 & -0.00000000001566 & $-0.99999999380945 \mathbf{i}$ & $-\mathbf{i}$
\end{tabular}


$10^{-13}$. Real zeros and spherical zeros did not show up. If $n$ is too large, say $n \approx 100$, then usually it is not any more possible to find all zeros of the companion polynomial by standard means (say roots in MATLAB) because the coefficients of the companion polynomial will be too large.

6. The quadratic case. We will specialize the given results to the quadratic case

$$
p_{2}(z):=z^{2}+a_{1} z+a_{0}, \quad a_{0}, a_{1} \in \mathbb{H}, a_{0} \neq 0 .
$$

We first repeat the results already given by Niven [13] in 1941. Then we will compare them with the foregoing theory. In all cases, we assume that $\Re a_{1}=0$. This simplifies some formulas, and there is no loss of generality since

$$
\begin{aligned}
\tilde{p}_{n}(u) & :=p_{2}\left(u-\frac{\Re a_{1}}{2}\right):=u^{2}+\left(a_{1}-\Re a_{1}\right) u+\frac{\Re a_{1}}{2}\left(\frac{\Re a_{1}}{2}-a_{1}\right)+a_{0} \\
& =: u^{2}+\tilde{a}_{1} u+\tilde{a}_{0}, \quad \Re \tilde{a}_{1}=0 .
\end{aligned}
$$

TheOREM 6.1. Let $p_{2}$ be given as in (6.1), and let $\Re a_{1}=0$.

1. If both $a_{1}, a_{0}$ are real (hence, $a_{1}=0$ ), then $p_{2}$ has either two different real zeros in $\mathbb{H}\left(a_{0}<0\right)$, or one spherical zero in $\mathbb{H}\left(a_{0}>0\right)$. The zeros in the first case are $\pm \sqrt{-a_{0}}$, the spherical zero is $[c]=\left\{z \in \mathbb{H}: z=h^{-1}\right.$ ch, $\left.h \in \mathbb{H} \backslash\{0\}\right\}$, where $c:=\sqrt{a_{0}} \mathbf{i}$.

2. If at least one of the coefficients $a_{1}, a_{0}$ is not real, then $p_{2}$ has either one or two isolated zeros in $\mathbb{H}$. It has one zero if

$$
2 \Re\left(a_{0} \overline{a_{1}}\right)=\left(2 \Re a_{0}+\left|a_{1}\right|^{2}\right)^{2}-4\left|a_{0}\right|^{2}=0 .
$$

It has two zeros, otherwise.

Proof. Niven (see Theorem 2, p. 658, of [13]).

The approach chosen here leads to the following: The companion polynomial for $p_{2}$ is

$$
q_{4}(x):=x^{4}+\left(2 \Re a_{0}+\left|a_{1}\right|^{2}\right) x^{2}+2 \Re\left\{a_{0} \overline{a_{1}}\right\} x+\left|a_{0}\right|^{2} .
$$

LEMma 6.2. The companion polynomial $q_{4}$ is a complete square if and only if the conditions of (6.4) are met.

Proof. Let $q_{4}(z)=\left(z^{2}+C z+D\right)^{2}=z^{4}+2 C z^{3}+\left(2 D+C^{2}\right) z^{2}+2 C D z+D^{2}$. Comparing with (6.5) yields $C=0, D^{2}=\left|a_{0}\right|^{2}$ and the conditions (6.4), hence, $q_{4}(z)=\left(z^{2} \pm\left|a_{0}\right|\right)^{2}$. If the conditions (6.4) are met, it is easy to see that $q_{4}$ is a complete square.

Lemma 6.3. Let the companion polynomial $q_{4}$ be a complete square, and let $q_{4}$ have two real zeros $r$ and $s$. Then $r+s=0$.

Proof. Let $q_{4}(z)=((z-r)(z-s))^{2}=\left(z^{2}-(r+s) z+r s\right)^{2}$. According to Lemma 6.2 we must have $r+s=0$.

As already noted in (3.4), real zeros come always in pairs. Thus, the existence of two different real zeros of $p_{2}$ always implies that $q_{4}$ is a complete square.

Corollary 6.4. Let $\pm r$ be two real zeros of $p_{2}$. Then, both coefficients $a_{0}, a_{1}$ of $p_{2}$ are real, and $a_{1}=0$ and $a_{0}<0$.

Proof. We have $r^{2} \pm a_{1} r+a_{0}=0$. If we subtract these two equations from each other, we obtain $2 r a_{1}=0$, thus, $a_{1}=0$. This implies $r^{2}+a_{0}=0$, hence, $a_{0}=-r^{2}<0$. 
TheOREM 6.5. Let $p_{2}$ be given as in (6.1) with $\Re a_{1}=0$. Then, there exists exactly one spherical zero $z \notin \mathbb{R}$ of $p_{2}$ if and only if $a_{0}, a_{1} \in \mathbb{R}$ and $a_{0}>0, a_{1}=0$. This zero is generated by $z=\sqrt{a_{0}} \mathbf{i}$.

Proof. A spherical zero $z \notin \mathbb{R}$ is characterized by $A(\Re z,|z|)=B(\Re z,|z|)=0$, where

$$
\begin{aligned}
& A(\Re z,|z|)=\alpha_{0} a_{0}+\alpha_{1} a_{1}+\alpha_{2} a_{2}=0 \cdot a_{0}+1 \cdot a_{1}+\Re z \cdot 1=a_{1}+\Re z=0, \\
& B(\Re z,|z|)=\beta_{0} a_{0}+\beta_{1} a_{1}+\beta_{2} a_{2}=1 \cdot a_{0}+0 \cdot a_{1}-|z|^{2} \cdot 1=a_{0}-|z|^{2}=0 .
\end{aligned}
$$

It follows that $a_{1} \in \mathbb{R}$ and thus, $a_{1}=0$, and because of $z \notin \mathbb{R} \Rightarrow z \neq 0$, we obtain $a_{0}=|z|^{2}>0$.

The last remainig case in which $q_{4}$ is a complete square is the following one:

$$
q_{4}(z)=((z-c)(z-\bar{c}))^{2}, \quad c \in \mathbb{C} \backslash \mathbb{R} .
$$

If at least one of the coefficients $a_{0}, a_{1}$ of $p_{2}$ is not real, both complex zeros $c, \bar{c}$ of $q_{4}$ are double zeros but produce the same isolated zero of $p_{2}$ (cf. formula (3.8)), and there are no other zeros of $p_{2}$. If $q_{4}$ is not a complete square and if at least one of the coefficients $a_{0}, a_{1}$ of $p_{2}$ is not real, there will be two isolated zeros of $p_{2}$. Thus, Niven's theory has been confirmed.

THEOREM 6.6. It is possible that the companion polynomial $q_{2 n}$ possesses pairs of nonreal, complex-conjugate zeros of multiplicity two and that the corresponding zeros of $p_{n}$ are isolated zeros.

Proof. We will present an example for this case.

Example 6.7. Let

$$
\hat{p}_{2}(\hat{z}):=\hat{z}^{2}+\hat{a}_{1} \hat{z}+\hat{a}_{0}, \text { where } \hat{a}_{1}:=\frac{\sqrt{3}}{3}(3,1,1,1), \hat{a}_{0}:=\frac{1}{2}(1,1,1,1) .
$$

Since the real part of $\hat{a}_{1}$ is not vanishing, we apply the transformation (6.2), namely $\hat{z}=z-\frac{\sqrt{3}}{2}$, and obtain

$$
p_{2}(z):=z^{2}+a_{1} z+a_{0}, \text { where } a_{1}:=\frac{\sqrt{3}}{3}(0,1,1,1), a_{0}:=-\frac{1}{4}(1,0,0,0) .
$$

For these coefficients the conditions of (6.4) are valid, and the companion polynomial is a complete square

$$
q_{4}(z)=\left(z^{2}+\left|a_{0}\right|\right)^{2}, \text { where }\left|a_{0}\right|=\frac{1}{4} .
$$

The only (isolated) zero of $p_{2}$ is

$$
-\frac{\sqrt{3}}{6}(0,1,1,1)
$$

which implies that the only (isolated) zero of $\tilde{p}_{2}$ is

$$
-\frac{\sqrt{3}}{6}(3,1,1,1)
$$

In the end, we will quote [15, Corollary 5, p. 388] of Pogorui and Shapiro. In order to understand the notation we give the following explanation: $\mathcal{R}_{n}$ is a polynomial of 
degree $n$, where the powers stand on the right side of the coefficients, correspondingly, $\mathcal{L}_{n}$ is a polynomial where the powers are located on the left side of the coefficients. The basic polynomial $\mathcal{F}_{2 n}^{*}$ is what we called the companion polynomial $q_{2 n}$.

"Given a polynomial $\mathcal{R}_{n}$ (or $\mathcal{L}_{n}$ ), there exist a one-to-one correspondence between its nonspherical zeroes and the pairs of the complex-conjugate zeroes of the basic polynomial $\mathcal{F}_{2 n}^{*}$ as well as a one-to-one correspondence between the spherical zeroes of $\mathcal{R}_{n}$ (or $\mathcal{L}_{n}$ ) and the pairs of complex-conjugate zeroes of multiplicity 2 of the basic polynomial $\mathcal{F}_{2 n}^{*}$."

According to the second part of this corollary, the polynomial $p_{2}$ defined in (6.7) should have a spherical zero, since the companion polynomial $q_{4}$ defined in (6.8) has a pair of complex-conjugate zeros of multiplicity 2. However, this is not the case, as we have shown in Example 6.7.

7. Summary of the algorithm. For finding the zeros of

$$
p_{n}(z):=\sum_{j=0}^{n} a_{j} z^{j}, \quad z, a_{j} \in \mathbb{H}, \quad j=0,1, \ldots, n, a_{n}=1, a_{0} \neq 0, n \geq 1,
$$

do the following steps:

1. Compute the real coefficients $b_{0}, b_{1}, \ldots, b_{2 n}$ of the companion polynomial $q_{2 n}$ by formula (3.2). Make sure that they are real.

2. Compute all $2 n$ (real and complex) zeros of $q_{2 n}$ (in MATLAB, use the command roots). Denote these zeros by $z_{1}, z_{2}, \ldots, z_{2 n}$ and order them (if necessary) such that $z_{2 j-1}=\overline{z_{2 j}}, j=1,2, \ldots, n$. If a specific $z_{2 j_{0}-1}$ is real, then it means that $z_{2 j_{0}-1}=z_{2 j_{0}}$.

3. Define an integer vector ind (like indicator) of length $n$, and set all components to zero. Define a quaternionic vector $Z$ of length $n$, and set all components to zero.

For $j:=1: n$ do

(a) Put $z:=z_{2 j-1}$.

(b) if $z$ is real, $Z(j):=z$; go to the next step; end if

(c) Compute $v:=\overline{A(z)} B(z)$ by formula (2.7), with the help of (2.3) to (2.5) on page 247 .

(d) if $v=0$, put ind $(j):=1 ; Z(j):=z$; go to the next step; end if

(e) if $v \neq 0$, let $\left(v_{1}, v_{2}, v_{3}, v_{4}\right):=v$. Compute $|w|:=\sqrt{v_{2}^{2}+v_{3}^{3}+v_{4}^{2}}$, put

$$
Z(j):=\left(\Re(z),-\frac{|\Im(z)|}{|w|} v_{2},-\frac{|\Im(z)|}{|w|} v_{3},-\frac{|\Im(z)|}{|w|} v_{4}\right) .
$$

end if

end for

The result of this algorithm will be an integer vector ind and a quaternionic vector $Z$, both of length $n$. If ind $(j)=1$, it signals that the complex number $Z(j)$ generates a spherical zero of $p_{n}$. In all other cases, $Z(j)$ will be an isolated zero of $p_{n}$. Though the quaternionic vector $Z$ has length $n$, the number of pairwise distinct entries may be smaller.

There are two delicate decisions to make in the above algorithm. In step 3(b) one has to decide whether $z$ is real. And in step 3(d) one has to decide whether $v$ is zero. Since a real zero of $q_{2 n}$ is always a double zero, and if one has not used the hints of the end of section 6 to improve on the precision of the real zeros, a test of the form 
$|\Im(z)|<10^{-5}$ is appropriate. In our experience, the test for $v=0$ can be carried out in the form $|v|<10^{-10}$. As already noted, steps 3(b), 3(d) occur in particularly constructed examples. In hundreds of random examples, we found that only step 3(e) occurred. But nevertheless, it would be wise to add a correction step in the zero finder for the companion polynomial $q_{2 n}$.

Acknowledgments. The authors would like to thank Ron Guenther, Oregon State University, Corvallis, OR, USA, for carefully reading this manuscript, resulting in several hints for improvement. Thanks go also to Swanhild Bernstein from Freiberg, Saxony, Germany, who brought the Klein quotation to our attention.

\section{REFERENCES}

[1] S. Eilenberg And I. Niven, The "Fundamental Theorem of Algebra" for quaternions, Bull. Amer. Math. Soc., 50 (1944), pp. 246-248.

[2] G. Gentili And D. C. Struppa, On the multiplicity of zeros of polynomials with quaternionic coefficients, Milan J. Math., 76 (2007), pp. 1-10.

[3] G. Gentili, D. C. Struppa, And F. Vlacci, The fundamental theorem of algebra for Hamilton and Cayley numbers, Math. Z., 259 (2008), pp. 895-902.

[4] G. Gentili and C. Stoppato, Zeros of regular functions and polynomials of a quaternionic variable, Michigan Math. J., 56 (2008), pp. 655-667.

[5] B. Gordon And T. S. Motzkin, On the zeros of polynomials over division rings, Trans. Amer. Math. Soc., 116 (1965), pp. 218-226.

[6] A. Gsponer and J.-P. Hurni, Quaternions in Mathematical Physics (2): Analytical Bibliography, Independent Scientific Research Institute report ISRI-05-05.26, 2008; also available online at http://arxiv.org/abs/math-ph/0511092v3.

[7] D. JanovskÁ AND G. Opfer, The classification and the computation of the zeros of quaternionic, two-sided polynomials, Numer. Math., 115 (2010), pp. 81-100.

[8] D. JANOVSKÁ AND G. OpFER, Givens' transformation applied to quaternion valued vectors, BIT, 43 (2003), Suppl., pp. 991-1002.

[9] F. KLEIN, Funktionentheorie in geometrischer Behandlungsweise, Vorlesung, gehalten in Leipzig 1880/81. Mit zwei Originalarbeiten von F. Klein aus dem Jahre 1882. Herausgegeben, bearbeitet und kommentiert von F. König, Teubner-Archiv zur Mathematik, Teubner, Leipzig, 1987, p. 296.

[10] T. Y. LAM, A first course in noncommutative rings, 2nd ed., Springer, New York, 2001, p. 385.

[11] D. LAURIE, Questions related to Gaussian quadrature formulas and two-term recursions, Applications and Computation of Orthogonal Polynomials, W. Gautschi, G. H. Golub, and G. Opfer, eds., Internat. Ser. Numer. Math., 131 (1999), Birkhäuser, Basel, Switzerland, pp. 133-144.

[12] S. De Leo, G. Ducati, and V. Leonardi, Zeros of unilateral quaternionic polynomials, Electron. J. Linear Algebra, 15 (2006), pp. 297-313.

[13] I. Niven, Equations in quaternions, Amer. Math. Monthly, 48 (1941), pp. 654-661.

[14] G. Opfer, Polynomials and Vandermonde matrices over the field of quaternions, Electron. Trans. Numer. Anal., 36 (2009), pp. 9-16.

[15] A. Pogorui And M. Shapiro, On the structure of the set of zeros of quaternionic polynomials, Complex Var. and Elliptic Funct., 49 (2004), pp. 379-389.

[16] S. Pumplün And S. WAlCher, On the zeros of polynomials over quaternions, Comm. Algebra, 30 (2002), pp. 4007-4018.

[17] R. Serôdio, E. Pereira, And J. VitóRia, Computing the zeros of quaternionic polynomials, Comput. Math. Appl., 42 (2001), pp. 1229-1237. 